\title{
SPECTRUM SENSING FOR COGNITIVE WIRELESS APPLICATIONS INSIDE AIRCRAFT CABINS
}

\author{
Christoph Heller, Christian Blümm, EADS Innovation Works, Munich, Germany \\ Stefan Bouckaert, Wei Liu, Ingrid Moerman, IBBT Ghent University, Belgium \\ Peter van Wesemael, Sofie Pollin, IMEC, Leuven, Belgium \\ Tomaz Solc, Zoltan Padrah, Jožef Stefan Institute, Ljubljana, Slovenia
}

\begin{abstract}
Wireless intra-aircraft communication is expected to be the enabler for more flexible avionic systems and the reduction of weight and cost in system installations. An alternative to the usage of a dedicated frequency band for wireless intra-aircraft avionics could be the usage of a virtually unregulated ISM band. Cognitive radio techniques could be used to increase system robustness in the likely case of interferences in this kind of frequency bands. A cognitive wireless cabin management system is discussed as a use-case for the validation of this approach. Using the mobile cognitive radio testbed of the FP7 project CREW, spectrum sensing experiments are carried out in a realistic aircraft cabin environment as a baseline for the development of suitable cognitive protocols and to record interference scenarios for the further system design.
\end{abstract}

\section{Introduction}

Future civil aircraft are expected to enclose a variety of systems based on wireless intra-aircraft communication. For air to ground links, wireless communication means are used since the early days of civil aviation, for instance in the fields of air traffic control and management. The intention to use wireless communication also within individual aircraft is motivated by the need to simplify system installation and reconfiguration, as well as by the need to reduce cost and weight of wired interconnections. Fields of application, where the usage of wireless intra-aircraft communication nowadays is evaluated, envisaged or even present, are flight test instrumentation (FTI), structural health monitoring (SHM) and cabin related system, such as the cabin management system (CMS). [1]

A general problem for the realization of such wireless intra-aircraft systems is the availability of a suitable frequency band. Commercial aircraft are intended to be used all over the globe, requiring a frequency band that is useable on a world-wide basis for this type of applications. The Wireless Avionics Intra-Communications (WAIC) initiative [2,3] - a consortium of aircraft manufacturers, avionics systems suppliers and aviation authorities - has prepared an agenda item for the World Radio Conference (WRC) of 2015 to ask for such a frequency allocation.

An alternative could be the usage of an Industrial/Scientific/Medical (ISM) frequency band, such as the popular band located at $2.45 \mathrm{GHz}$ in $\mathrm{S}$ band or the one centered at $5.8 \mathrm{GHz}$ in C-band. The previously outlined applications doubtlessly can be classified as industrial applications, giving them the rights to use an ISM frequency. The inherent problem of using an ISM frequency, even mentioned in the corresponding radio regulation of the International Telecommunications Union (ITU), is that equipment operating in these bands must accept harmful interference with other devices operating in the same band [4]. For an intra-aircraft system, interferences might originate from electronic equipment brought on board by passengers, such as active Wi-Fi or Bluetooth connections from laptops, mobile phones or wireless headphones.

The idea forming the basis of this paper is to use cognitive radio techniques to increase the robustness of a safety related wireless system operating in a shared frequency band. Following the classical principles of cognitive radio [5], spectrum sensing will be performed to monitor the actual spectrum usage. Based on the current interference scenario, a cognitive unit then has to make a decision about what portion of the frequency shall be used by the wireless aircraft system to optimally meet its performance goals regarding data throughput and robustness. Finally, the wireless adaptors of the system will be reconfigured to execute the decision of the cognitive unit. These tasks are periodically repeated to 
dynamically respond to dynamically changing interference scenarios.

This paper focuses on the discussion of the CMS as a use-case system to evaluate, whether cognitive radio can improve communication performance in an unregulated frequency band. It presents a spectrum sensing experiment that has been performed in a realistic mock-up of an aircraft cabin, using the mobile cognitive radio testbed developed in the scope of the European FP7 research project CREW (Cognitive Radio Experimentation World) [6]. The experiment should support the conceptual design of the cognitive wireless CMS by answering questions such as whether to use distributed or centralized spectrum sensing and what kind of propagation effects have to be expected resulting from the metallic environment of the aircraft cabin. It should also serve to record meaningful interference scenarios for the further development of the cognitive system.

The remainder of this paper is structured as follows. First of all, the CMS is discussed as a potential use-case for a cognitive wireless aircraft system. The research project CREW is presented in the following section, focusing on the mobile cognitive radio testbed that has been used for the spectrum sensing experiments discussed in this paper. Then the experiment itself and its results are presented. The paper concludes with a discussion of the experimentation results and by outlining the next steps to a prototype of the cognitive wireless CMS.

\section{Potential Use-Case Systems}

FTI, SHM and cabin systems can be identified as possible candidates for wireless intra-aircraft communication, where flexibility can be increased and cost can be decreased by omitting cables and harnesses for data transmission. Before discussing the CMS, a rough outline about wireless FTI and SHM applications is given.

For FTI applications, the test aircraft is equipped with a great number of sensors, measuring for instance structural loads and strains, acceleration or aerodynamic streams. The sensors are located inside the aircraft and also at critical positions outside the pressurized compartments. Even if smart sensors with digital bus interfaces are used, a large number of cables is required to connect the different sensors to data collectors and recording devices. Wired connections crossing the hull of the aircraft are particularly critical, since these feedthroughs have to be hermetically sealed and able to withstand the occurring pressure differences during flights. A wireless FTI installation would significantly simplify the sensor installation, coming along with reduced preparation times and cost. [1]

The field of SHM is similar as FTI, with the difference that flight test sensors are only used in dedicated test aircraft, whereas health monitoring sensors are used in 'normal' aircraft being on duty for airlines. Their purpose is to monitor structural loads, abrasion or wear of critical components for effective maintenance and replacement of worn out components. Besides the simplified installation of wireless SHM systems, the reduced weight results in lower fuel consumption and thus in lower operating cost of the aircraft. [1]

In the field of cabin systems, the CMS is a typical candidate for the deployment of wireless system communication. Functions handled by this system comprise reading lights, passenger calls for flight attendants (PAX calls), visual signs (e.g. "Fasten Seatbelts" or "No Smoking") and audio announcements from crew personnel to passengers [7]. Passengers mainly get in touch with the system via the Passenger Service Units (PSU), panels comprising loudspeaker, reading lights, signs and pushbuttons to operate the device (cf. Figure 1). One PSU generally is mounted in the aircraft cabin above each group of seats in a dedicated service channel together with the air-conditioning outlets and the oxygen masks.

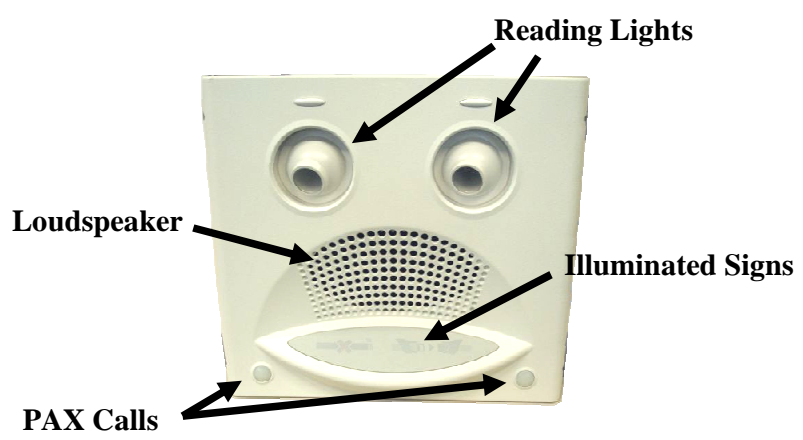

Figure 1. Passenger Service Unit (PSU)

There are mainly two motivations to use wireless communication for the CMS. The first one is 
increasing flexibility for reconfiguring the aircraft cabin. Airliners demand the ability to completely reconfigure the seat layout of the cabin of future aircraft during turn-around times at the gates, which typically are in the range of 30 to 90 minutes, depending on the size of the aircraft [8]. This means that according to the passenger volumes in firstbusiness- and economy-class, the number of seats in each class should be adaptable to optimize ticket revenues. For this purpose, the numbers of seats and also their positions in the cabin have to be as flexible as possible. For the CMS this implies that the numbers of PSUs and also their positions in the cabin have to be flexible. When switching between classes, it also might be necessary to change a PSU servicing a group of two seats against a PSU servicing three seats.

Such flexibility is difficult to attain in a wired system. It would require a harness with many redundant connectors for all possible PSU locations and additional meters of cable to be able to move the devices to different locations within the service channel. In a wireless CMS, the problem of cabling can be significantly reduced. Of course the PSUs still have to be connected to a power source, but this can be accomplished by using the metallic rails of the service channel the PSUs are mounted at as power feeds. Power connections generally are much less critical than those for high-speed communication, since no controlled impedances are required [9]. A significant part of the system harness including complex and difficult to handle connectors could become obsolete when having a wireless CMS.

Besides the reductions in system cost, complexity and weight, a wireless CMS would bring the benefit of enabling new functions that are not possible in a wired system. The main interface of the CMS towards the aircraft crew is the Flight Attendant Panel (FAP), a touch-screen device generally installed next to the doors of the aircraft that allows flight attendants to control the cabin functions (cf. Figure 2). In a wireless CMS, the FAP could become a mobile device, for instance included in a small handheld tablet computer, reducing the crew workload by enabling them to control the cabin from everywhere inside the aircraft. Announcements from attendants to the passengers as well as communication between crew members could be enabled with wireless headsets in an easier and more flexible way having a wireless infrastructure to connect them with. $[7,10]$

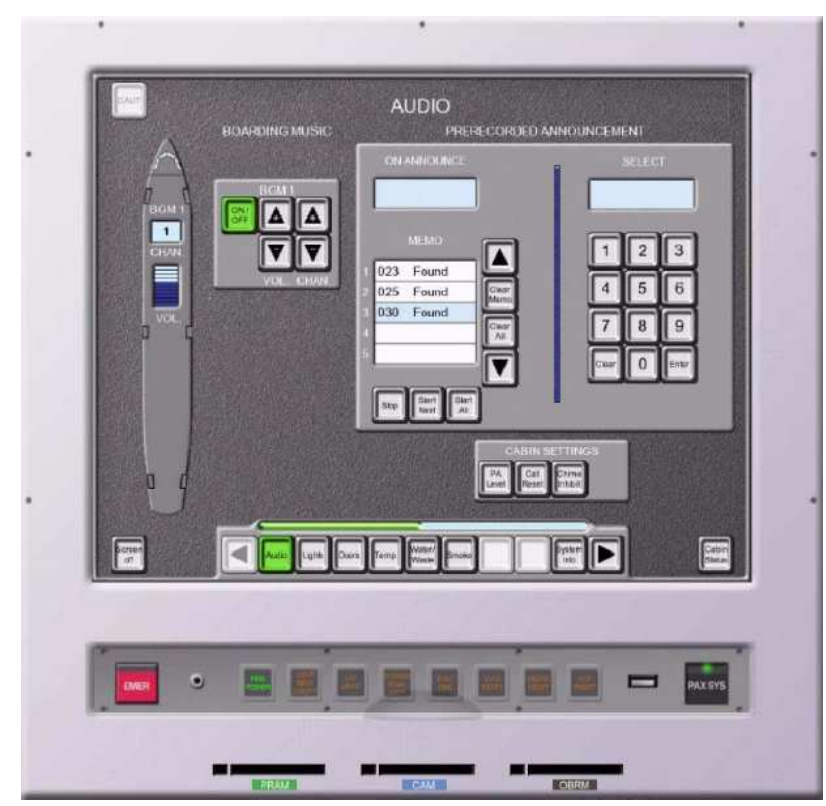

\section{Figure 2. Exemplary Screenshot of Flight Attendant Panel (FAP)}

The CMS thus is a good candidate system to evaluate the introduction of wireless intra-aircraft communication. As for all such systems, frequency regulation nowadays is an unsolved issue. When operating the system in a virtually unregulated frequency band, such as an ISM band, availability might not be guaranteed in case of interferences. For the wireless CMS this could become critical, since its functions are to a certain extent safety relevant. The U.S. Federal Aviation Department has defined general categories for failure conditions [11]. Base on them the Radio Technical Commission for Aeronautics Inc. has defined five Design Assurance Levels (DAL), that apply for hardware [12] and software [13] design. The CMS comprises functions rated as DAL-D ("minor" failure impact) and DAL-C ("major" failure impact), as summarized in Table 1. 
Table 1. Criticality and Data Rate Requirements of Selected CMS Functions [14]

\begin{tabular}{|c|c|c|}
\hline CMS Function & DAL & Data Ra \\
\hline $\begin{array}{l}\text { Pre-Recorded Audio } \\
\text { Announcements }\end{array}$ & $\mathrm{D}$ & \multirow{3}{*}{$\begin{array}{c}6.2 \mathrm{Mbit} / \mathrm{s} \\
\text { (sum of all } \\
\text { audio } \\
\text { channels) }\end{array}$} \\
\hline $\begin{array}{l}\text { Evacuation/Emergency } \\
\text { Announcements }\end{array}$ & $\mathrm{C}$ & \\
\hline CREW Interphone & $\mathrm{C}$ & \\
\hline Reading Lights & $\mathrm{D}$ & \multirow{3}{*}{$\begin{array}{c}<1 \mathrm{Mbit} / \mathrm{s} \\
\text { (sum of all } \\
\text { control } \\
\text { channels) }\end{array}$} \\
\hline PAX Calls & $\mathrm{C}$ & \\
\hline Sign Contro & $\mathrm{C}$ & \\
\hline
\end{tabular}

For a DAL-D system, the probability of failure must not exceed the range $10^{-3} \ldots 10^{-5}$ per flight hour, the corresponding range for DAL-C systems is $10^{-5}$ $\ldots 10^{-7}$ [11]. Reaching this robustness in a wireless system operating in a frequency band where interferences with other systems are more than likely is a challenge. Cognitive radio could be an option to avoid interferences or reduce their negative impact on system performance. To evaluate this approach, the CREW mobile cognitive radio testbed comes into play.

\section{The Cognitive Radio Project CREW}

The Cognitive Radio Experimentation World (CREW) project, funded under the European FP7's Future Internet Research and Experimentation (FIRE) program, establishes a federation of European cognitive radio testbeds that aims at facilitating experimental research. Wireless testbeds from different partners located at different European countries are interconnected via the Internet to allow users to run their cognitive radio experiments on individual or virtually interconnected testbed configurations. [6]

\section{The Federated Cognitive Radio Testbed}

In its current implementation, depicted in Figure 3, CREW federates a software defined radio testbed at Trinity College Dublin (Ireland), a heterogeneous ISM wireless testbed at IBBT in Ghent (Belgium), a sensor network testbed at TU Berlin (Germany), an outdoor heterogeneous ISM/TV White Spaces testbed at Josef Stefan Institute in Ljubljana (Slovenia), a spectrum sensing platform developed at imec in Leuven (Belgium), and an Long Term Evolution
(LTE) testbed at TU Dresden (Germany). Additional partners can join the federation to conduct experiments on the testbeds and/or integrate their own cognitive radio platforms or cognitive software solutions in the existing testbeds.

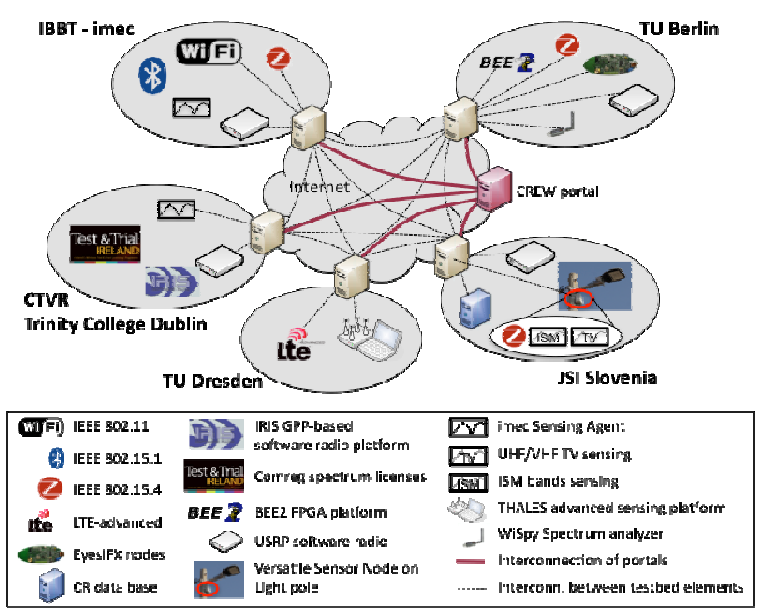

Figure 3. Overview of the CREW Testbed

A common portal has been established to all testbeds in the federation. This portal gives a comprehensive description of the individual testbeds and the functionalities of the federated testbed and further provides clear guidelines on how to access and use the federated testbed. By linking together software and hardware components of multiple partners, advanced cognitive components (such as spectrum sensing agents) can be generated. Data is shared among the testbeds and components using common application programming interfaces (API) and specific common data formats. A benchmarking framework for cognitive radio and network experiments offers automated procedures for configuring and running experiments following welldefined performance evaluation methodologies, thus enabling comparison between subsequent developments or competing cognitive solutions.

Three primary operating modes for the federation are supported:

- In mode 1 , experimenters can utilize the common CREW portal to access an individual testbed in the federation.

- Mode 2 enables the hosting of nodes from one testbed in another and the creation of new nodes from combinations of hardware and software 
components developed in different testbeds in the federation.

- Mode 3 defines the sequential use of testbeds, capturing data and behaviors observed in one testbed and replaying those in a different testbed, emulating the joint operation of multiple testbeds.

More information on CREW, the federated testbed and the equipment available at the certain facilities can be found in [6].

\section{The Mobile Cognitive Radio Testbed}

For certain types of use-cases and experiments, the static installation of the testbed equipment turns out to be rather unhandy. These are experiments, where the environment is assumed to have major impact on the propagation of wireless signals and their perception at receivers and spectrum sensing equipment. For such scenarios, parts of the equipment can be relocated to perform experiments in the specific environment. The common data format of CREW allows re-integration of the obtained results into the fixed testbed installation to continue experimentation with the full set of equipment. The mobile testbed also supports the setup of a temporary portal server for remote access, automated scheduling of experiments and benchmarking, if this is required for the specific use-cases.

For the wireless CMS, the interference scenario created by interfering passenger devices is a crucial point that is assumed to be strongly influenced by the metallic structures surrounding the cabin. If a set of representative interference scenarios can be captured with the mobile testbed in a realistic environment, protocols for cognitive communication can be further developed and tested based on these recordings. This capturing as well as some related experiments are described in the following section of this paper.

\section{The Spectrum Sensing Experiments}

This section describes the setup, the execution and evaluation of the spectrum sensing experiments in a realistic aircraft cabin environment using the CREW mobile cognitive radio testbed.

\section{Test Environment}

The experiments have been performed in an Airbus A340 mock-up located on the premises of
EADS Innovation Works in Munich, Germany. The layout and approximate dimensions of the mock-up are shown in Figures 4 and 5. The cabin comprises 13 groups of two business-class seats. All the inside materials (e.g. the lining, seats, overhead compartments, ceiling panels) are original equipment, whereas the outside hull and all structural parts consist of wood. To get a more realistic environment, these wooden parts have been coated with aluminum foil. Since the skin depth in aluminum for the investigated frequency band of $2.4 \mathrm{GHz}$ is in the range of $1 \ldots 2 \mu \mathrm{m}$, massive metallic elements would have the same effect on electromagnetic wave propagation [9]. With $6.6 \mathrm{~m}$, the length of the mockup is rather limited and its ends are open. This is assumed to be the biggest deviation from a real aircraft cabin, considering the impact on electromagnetic field propagation. Measurements taken in the middle of the cabin segment are assumed to be most realistic.

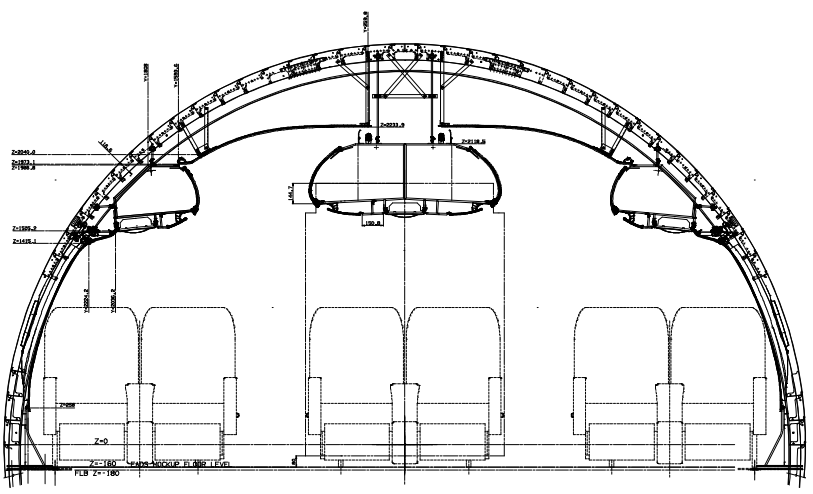

Figure 4. Cross Sectional View of Aircraft Cabin Mock-Up

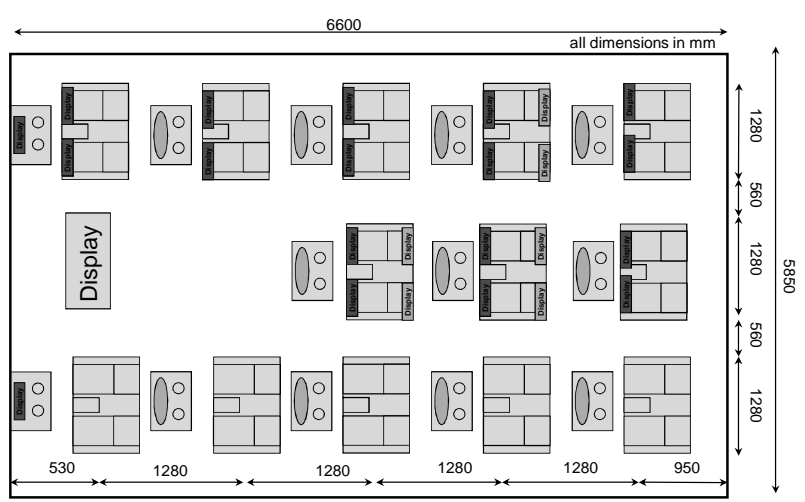

Figure 5. Floor Plan of Cabin Mock-Up 


\section{Equipment Selection and Installation}

For spectrum sensing in non-cooperative systems, three different approaches are distinguished: Energy detection is the simplest and less sensitive method that is based on measuring the signal energy within a certain frequency range and compares this energy level with a defined threshold. The second method, cyclostationary feature detection, determines the autocorrelation function of the signal to find periodical components inside the signal. The third and most complex approach is based on matched filtering with a filter designed according to the signal to be detected. [15]

The selection of sensing equipment from the available devices of the crew testbed is closely coupled to the use-case itself. Since the ISM band considered for the system is virtually unregulated, it is not known which type of equipment and in particular which type of signal might interfere with the CMS. Spectrum sensing therefore has to be based on energy detection, which is able to detect any sufficiently strong interfering signal without requiring knowledge of the signal to be detected. The sensing equipment installed in the cabin mock-up as part of the mobile testbed is as follows:

- Imec advanced sensing agents (based on the flexible Scaldio RF chips and Matlab post-processing $[16,17])$

- USRP2 hardware platforms with WBX frontends. Captured baseband samples are post-processed on a Matlab computer $[16,17,18]$

- VSN nodes based on CC2500 series transceivers [19,20]

One device of each type of sensing equipment has been installed at the ceiling in the center of the cabin in order to represent a wireless PSU. Another instance has been installed on a food serving trolley to represent a wireless FAP. The trolley has been placed in the right aisle of the cabin.

Additionally, two w-iLab.t nodes [21] from IBBT have been installed on the front left and front right seats. They are used to create interfering Wi-Fi signal transmissions, emulating active Wi-Fi adaptors of passengers' laptops.

All equipment is connected to external control, signal processing and recording devices using either
Ethernet or Universal Serial Bus (USB) connections. Due to the great flexibility and modularity of the CREW mobile testbed, the complete experiment setup did not take longer than approximately two hours.

\section{Experiment Execution}

The w-iLab.t nodes located on the front seats are used to create a pre-defined interference scenario, corresponding to uncontrolled passenger Wi-Fi activity. The transmitted signals are IEEE $802.11 \mathrm{~g}$ compliant. While the node located on the right seat is continuously transmitting on Wi-Fi channel 11 , the node on the left seat switches between channels 1,6 , $3,10,4$ and 11 (in this order). The experiment is repeated for strong continuous signal transmissions and weaker signal transmissions consisting of shorter bursts. While the sensing equipment located at the cabin ceiling stays at its fixed position, the trolley with the sensing devices is moved from the front of the cabin to its other end and back during approximately 90 seconds. All spectrum data is collected and recorded for further post-processing and analyses.

\section{Results}

Some exemplary spectral captures for the previously described scenario are shown in Figures 6 and 7. For the strong and continuous signals, all equipment, even the one based on simple wireless transceivers such as the VSN, were able to reliably sense the spectrum for both sensor locations.

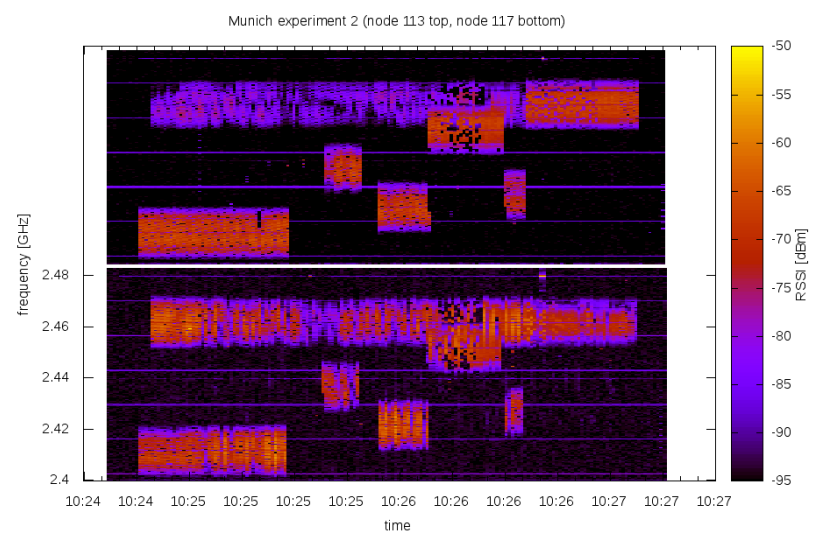

Figure 6. Spectrum Seen by VSN Nodes at Ceiling (Top) and on Trolley (Bottom) for Strong Continuous Signals 
For the weaker signal scenario, even the rather high-end Scaldio transceiver has some difficulties to detect the signal (cf. Figure 7). Here also some frequency selective fading effects become visible in the non-constant amplitude levels of the spectral parts occupied by the Wi-Fi signals. This effect is probably caused by signal reflections at the metallic structure of the cabin. The rather wide-band Wi-Fi signals can still be recognized, whereas it could become difficult to reliably detect narrow-band signals, in case they spectrally coincide with a deep fade.

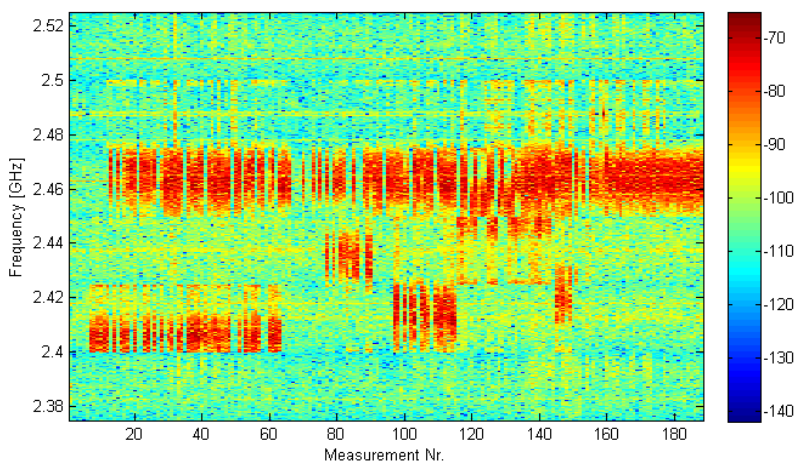

Figure 7. Spectrum Seen by Imec Sensing Agent on Trolley for Weak and Bursty Signals

Besides these frequency selective fadings, there are no real slow fading or attenuation effects visible, not even for the trolley, which varies its distance to the transmitting nodes during the experiment. It is assumed that this behavior originates from a kind of wave-guiding effect of the metallic outside wall, so that the power decay with distance is less than in free space. Comparative measurements with the same setup in a non-metallic environment could prove these assumptions.

The focus of this paper is on the presentation of the use-case system and the concept of using the mobile CREW testbed. A comparison of the sensing performances of the different spectrum sensing solutions available in the CREW federation can for instance be found in $[16,17]$.

\section{Conclusion and Next Steps}

This paper has presented the wireless CMS as a potential use-case for the improvement of system robustness by using cognitive radio techniques in a wireless system operating in a congested spectrum with likely and uncontrollable interferences. It has been shown that the CREW mobile cognitive radio testbed can easily be deployed in special wireless environments, such as an aircraft cabin, in order to perform cognitive radio experimentation with realistic electromagnetic field propagation and environmental effects. The spectrum sensing experiments described in this paper already give some hints for the proper design of a cognitive wireless aircraft system. Frequency selective fading effects, likely caused by the metallic environment, make it impossible for single spectrum sensing devices to reliably detect narrow-band interferers. A robust cognitive intra-aircraft system thus should be based on a distributed spectrum sensing system, where multiple sensing devices located at different position jointly perform the sensing task, reducing the risk of missing a signal due to fading. The waveguiding effect of the metallic cabin leads to smaller signal attenuations than in free-space, so that interfering signals devices might disturb subsystems of the wireless CMS at a greater extent.

The interference scenarios captured in the aircraft cabin now can be used for further developing the cognitive wireless CMS. In a next step, a cognitive unit is required that decides about the spectrum occupancy and the presence of interferers. It then has to make a decision about what frequency range to be used by the wireless CMS. Either the center frequency of the CMS communication is shifted to a non-disturbed spectrum portion, or - in the presence of narrow-band interferers, parts of the CMS spectrum can be masked out, as demonstrated in figure 8.

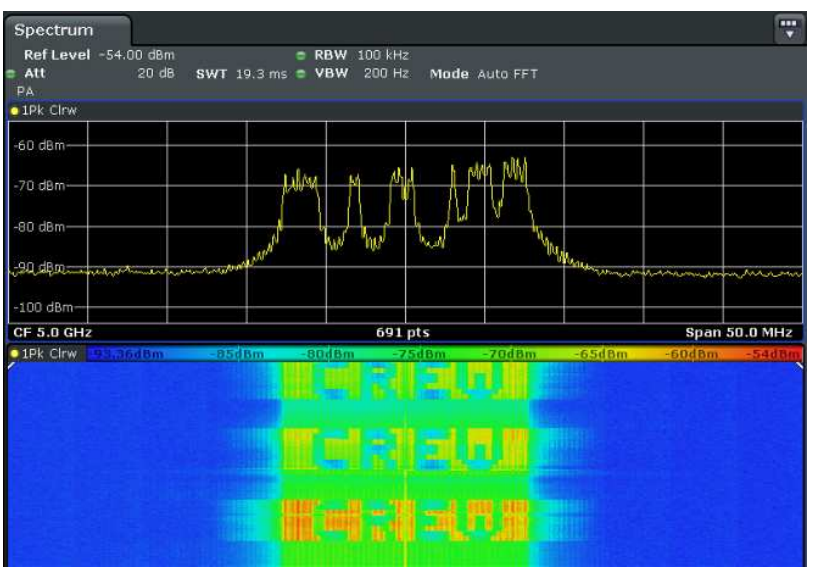

Figure 8. Demonstration of Spectrally Flexible OFDM Waveform 
In this example, particular subcarriers of an Orthogonal Frequency Division Multiplex (OFDM) waveform can be masked to be immune against narrow-band interferers. To demonstrate this flexibility, the word "CREW" has been written in the waterfall plot of a spectrum analyzer.

The next steps, accordingly, are to select an appropriate decision algorithm and connect it with the spectrally flexible wireless transmission system currently under development. Finally, some comparative measurements have to be done to quantify the gain in robustness achievable by using cognitive radio methods in the wireless CMS. For the development of the cognitive decision algorithms, the CREW federated cognitive radio testbed is assumed to provide good support and the required equipment. For a later system validation in the realistic aircraft environment, the mobile testbed could again be useful.

\section{References}

[1] Blanckenstein, Johannes, Jirka Klaue and Holger Karl, 2012, Energy efficient clustering using a wakeup receiver, in proceedings of the $18^{\text {th }}$ European Wireless Conference EW2012, Poznan, Poland

[2] Cramer, Joseph, 2012, Agenda Item 1.17 Wireless Avionics Intra-Communications (WAIC), presentation given at workshop on preparations for WRC-15

[3] ITU (International Telecommunications Union, 2010, Technical characteristics and operational objectives for wireless avionics intracommunications (WAIC), report ITU-R M.2197

[4] ITU (International Telecommunications Union), Radio Regulation RR 5.150

[5] Arslan, Hüseyin, 2007, Cognitive Radio, Software Defined Radio, and Adaptive Wireless Systems, Springer

[6] CREW, Cognitive Radio Experimentation World, project website: www.crew-project.eu

[7] Leipold, Frank, Dimitri Tassetto and Sergio Bovelli, 2011, Wireless in-cabin communication for aircraft infrastructure, Springer US, Journal of Telecommunication Systems, pp. 1-22.
[8] ACARE (Advisory Council for Aeronautic Research in Europe), 2008, Strategic Research Agenda

[9] Johnson, Howard, Martin Graham, 2003, HighSpeed Signal Propagation, Prentice Hall

[10] ETSI (European Telecommunications Standards Institute), 2009, Electromagnetic compatibility and Radio spectrum Matters (ERM); System Reference Document; Technical characteristics for airborne Ultra-WideBand (UWB) applications operating in the frequency bands for $3,1 \mathrm{GHz}$ to $4,8 \mathrm{GHz}$ and $6 \mathrm{GHz}$ to $8,5 \mathrm{GHz}$, technical report ETSI TR 102834 V1.1.1

[11] U.S. Department of Transportation, Federal Aviation Administration, Advisory Circular 25-1309$1 \mathrm{~A}$

[12] Radio Technical Commission for Aeronautics Inc., Design Assurance Guidance for Airborne Electronic Hardware, DO254

[13] Radio Technical Commission for Aeronautics Inc., Software Considerations in Airborne Systems and Equipment Certification, DO178B

[14] EUWB Integrated Project, 2008, Deliverable D8a.1: Scenario description for public transport applications

[15] Subhedarl, Mansi, Gajanan Birajdar, 2011, Spectrum sensing techniques in cognitive radio networks: a survey, International Journal of NextGeneration Networks (IJNGN) Vol.3, No.2, pp. 3751

[16] Wesemael Van, Peter, Sofie Pollin, Eduardo Lopez, Antoine Dejonghe, 2011, Performance Evaluation of Sensing Solutions For LTE and DVB$\mathrm{T}$, in proceedings of DySpan 2011, Aachen, Germany

[17] Finn, Danny et al., 2011, Experimental Assessment of Tradeoffs among Spectrum Sensing Platforms, in proceedings of WiNTECH 2011, Las Vegas, Nevada

[18] http://www.ettus.com/

[19] http://www.crew-project.eu/vsn

[20] Fortuna, Carolina, Zoltan Padrah, Thomaz Zolc, 2012, CREW - LOG-a-TEC and JSI Sites, in proceedings of FIRE Hands-on, Aalborg, Denmark

[21] http://www.crew-project.eu/wilabt 


\section{Acknowledgements}

The research leading to these results has received funding from the European Union's Seventh Framework Programme FP7/2007-2013 under grant agreement $\mathrm{n}^{\circ} 258301$ (CREW project).

31st Digital Avionics Systems Conference

October 14-18, 2012 
Quick Links

- Final Program (pdf)

- Photographs

- Committee

Sponsors

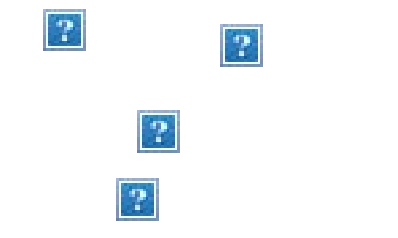

Exhibitors
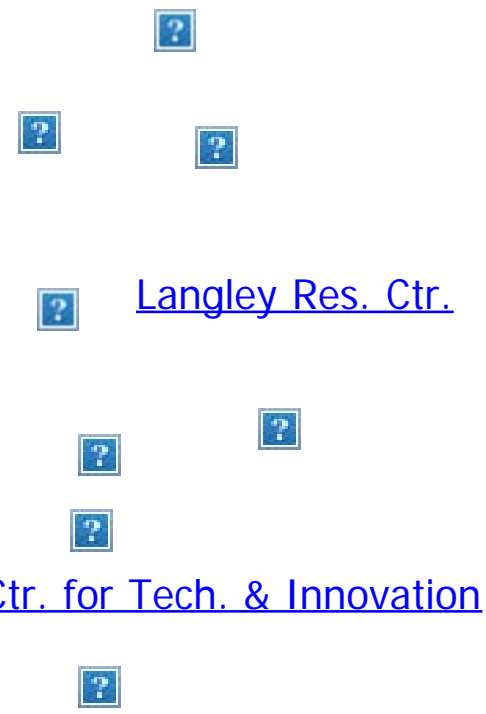

\section{Crowne Plaza Williamsburg, Williamsburg, VA October 14-18, 2012}

\section{General Chair's Thank You}

Hello Again Ladies and Gentlemen,

Thanks for visiting the DASC and checking-in here for the Chair's Summary. You participated in the 31st DASC or wish you had, presumably, because you are a technical professional with a keen interest in advanced aviation concepts and designs.

As autumn is upon us, we reflect on the past year and how we faired in Williamsburg. The 17th century capital of colonial Virginia is where our efforts in reviewing the history of aerospace electronics and its projection into the future of avionics culminated. If we learned nothing else from our review of history, let's walk away with the renewed commitment to learn more. Let's continue to realize that we "don't know" everything about our history that will help us in future avionics decisions. Also, let's make no mistake - the "right" approach towards the next 100 years of avionics will positively influence all of mankind. I believe that we are part of the solution and I have personally been honored to briefly contribute in leading us down this path for this past year.

With over 300 participants, we are certain that the environment was produced at the DASC where evolving electronics and other engineering advances have been shared that will enhance flight.

The DASC organized a technical paper program, a set of useful tutorials, and student-focused activities. The authors submitted abstracts and papers that covered the gambit of Flight Deck Systems; Air Traffic Management (ATM); Communications, Navigation and Surveillance (CNS); Unmanned Aircraft Systems (UAS); Systems and Software Engineering; Information Management, Networks, and Architecture; and Verification and Validation of Complex Systems. We evaluated the research papers and although all were superb, identified those we believed warranted award recognition. Throughout the conference, attendees and authors exchanged views that will only enhance future advances in aviation. The tutorials provided systems knowledge needed for HW/SW design assurance and methods for managing faults; standards for real time operations; systems engineering perspectives in avionics and space; surveillance and collision avoidance; synthetic and enhanced vision; applicable network and wireless; future navigation; acquisition processes for special avionics and systems; and 
review of digital avionics systems and architectures. This year we introduced a high school student competition "Avionics

Competition/Challenge", and we were all pleasantly surprised at the quality of work presented by these young people. Theirs was a very popular booth at the exhibition. Thanks to all our sponsors and exhibitors in making our venue memorable.

We introduced an Interactive Workshop where we encouraged more rapid advances in current applicable technologies. We set the goal to: "Define today's avionics programs based on the integration of 7 scenarios for what we think the air and space flight electronics will look like in 2112." As a result of the Workshop, very little is "off the table" as far as what can be accomplished over the next 100 years. The question becomes, will we actively, safely, and efficiently pursue the tough challenges set before us?

Through special Sessions/Panels, we were fortunate to hear about and see presentations on specific avionics issues while having questions answered by leaders in the field. The kickoff Plenary Session gave us a professional rendition of the history of avionics through the encompassing presentation by our Smithsonian Curator; NASA HQ's Director for Aviation Safety Research linked our current activities to what we think the future looks like; the SR-71 pilot and program manager from L-3 ComCept opened our eyes to what can be possible when engineering is brought to bear on welldefined aviation goals and objectives - this gave us a lesson to take to the future; the President for sales of Bombardier gave us perspective on what the 20 year future in business jet aircraft looks like; the VP of Technology for Rockwell Collins gave us a technically sound near/far term rendition of technical challenges we should be cognizant of; and the Deputy FAA Deputy Associate Administrator for Aviation Safety gave us the realistic state of current and future challenges facing government execution of aviation programs. Our final Lunch Panel gave us a riveting account of the evolution and future projection of avionics from the Mitre Corporation's Director of CNS Engineering \& Spectrum; the FAA's Acting Director of Engineering Services; Gulfstream Aerospace's Director of Advanced Cockpit Programs; NASA Langley Research Center's Director for Aeronautics Research; and Lockheed Martin's Director of Aeronautical Systems. The Virginia Air and Space Museum provided the perfect backdrop for our Air Force Historian (ret) to give the concluding historic presentation. We appreciated the knowledge shared by him and all these executives.

It is my belief that we will soon see advances in fields such as quantum mechanics, plasmonics, nano-scale gravitational field management, associated electromagnets, and phonon/photon characterization for digital applications. We'll also see revolutionary material electronics in flight surfaces, controls, and power/ propulsion management schemes. I hope you all are a part of these advances and use your technical expertise in refining these and other related physical concepts and principles while safely moving digital aerospace electronics forward. We have come a long way since vacuum tubes and terrain following navigation.

I am venturing to speak here for our authors; students; educators; special, track, and session chairs; exhibitors; sponsors; special event speakers; and the DASC committee when I say that we are satisfied that the 31st DASC stimulated the thought required to carry us through the 21st century and beyond. As a 32nd DASC contributor in aviation or aerospace electronics you will help influence the collaboration of efficiencies and safety throughout the industry next October. Set your calendars for Sunday the 
10th through Thursday the 14th, now. We look forward to your participation in the October 2013 DASC.

Email comments on this web site to the Web Manager. This file was last modified on EDT. Copyright @ 2012 Digital Avionics Systems Conference 